\title{
HYSTERETIC BEHAVIOR IN AN Fe-BASED SHAPE MEMORY ALLOY UNDER TENSILE/COMPRESSIVE CYCLIC THERMOMECHANICAL LOADING
}

\author{
Fumihito Nishimura, Noriko Watanabe and Kikuaki TANAKA \\ Department of Aerospace Engineering \\ Tokyo Metropolitan Institute of Technology \\ Hino, Tokyo, 191, Japan
}

\begin{abstract}
The thermomechanical hysteretic behavior in an $\mathrm{Fe}-9 \% \mathrm{Cr}-5 \% \mathrm{Ni}-14 \% \mathrm{Mn}-6 \% \mathrm{Si}$ polycrystalline shape memory alloy is experimentally investigated under tensile and compressive cyclic thermomechanical loading. Similar to the other shape memory alloys, such as $\mathrm{Ti}-\mathrm{Ni}$ and $\mathrm{Cu}$-based alloys, the $\mathrm{Fe}$-based shape memory alloy also exhibits the cyclic effect which appears macroscopically as the change in the hysteresis loop with cycling. The convergence to a limit loop is, however, not observed in the present alloy. The cyclic effect on the residual strain, the transformation strain and the martensite start stress is explained by the two metallurgical processes; the accumulation of the perfect dislocations and the shape irreversibility in the reverse transformation.
\end{abstract}

Key words: Fe-based shape memory alloy, Cyclic thermomechanical loading, Tension and compression, Hysteretic behavior.

\section{INTRODUCTION}

Fe-based shape memory alloys have been developed [1-5] as a promising alternative to the conventional shape memory alloys such as $\mathrm{Ti}-\mathrm{Ni}$ and $\mathrm{Cu}$-based alloys. Due to the attractive advantages of the high strength, good workability and the low cost, they are expected to be used as the large shape memory devices like the fastener of pipes [6], while the other shape memory alloys are used as the small devices in the form of the wires, coils or the thin plates $[7,8]$. The technology of careful alloying and the heat treatment has realized the various types of Fe-based alloys which exhibit, in addition to the desirable high shape memory properties, the special functions such as the corrosion resistance, machinability and so on [9].

The shape memory effect in many Fe-based alloys is associated with the stress-induced transformation from the parent $(\gamma)$ phase to the $\varepsilon$-martensite phase and its reverse transformation during heating. The reversible transformation is realized by the reversible change in the stacking sequence between $\mathrm{ABCA} \ldots(\gamma)$ and $\mathrm{ABAB} \ldots(\varepsilon)$, which is caused by the reversible motion of the Shockley partial dislocations. The morphological aspect of the transformation is revealed to progress as follows [10,11]: At the early stage of loading the thin $\varepsilon$-martensite, roughly of the order of $1 \mathrm{~nm}$ in thickness, are formed uniformly in the specimen as the stacking faults. Experiments show that not more than two types (directions) of $\varepsilon$-martensite are observed in a grain. The thickness of some $\varepsilon$-martensite becomes larger upon further loading. This is attributed not to the growth of the $\varepsilon$-martensite plates but to the continuous nucleation of the new thin $\varepsilon$-martensite plates with the same direction beside the original ones. Thus a cluster structure of the $\varepsilon$-martensite plate is formed in the specimen. This formation of the cluster does not progress at all $\varepsilon$-martensite plates simultaneously. The fraction of martensite is, therefore, microscopically inhomogenous in the specimen. It should be noted that one parent phase plate is always laminated between the two $\varepsilon$-martensite plates, meaning a full transformation is not achieved in a global sense although the specimen looks to be fully covered by the $\varepsilon$-martensite plates.

When the alloy is subjected to the cyclic thermomechanical loading, the phase interfaces propagate forward and backward repeatedly, resulting in the cyclic, but not always reversible, change in the microstructures. The pileup of the dislocations are observed at the microscopic defects scattered in $\mathrm{Cu}$-based alloys, inducing there the microscopic residual stresses/strain and the stabilized martensite which does not take part in the subsequent transformations [12]. The macroscopic transformation and deformation response of the shape memory alloys thus exhibits a strong cycle-dependence $[13,14]$. The cyclic effect has some negative effect on the practical use of the shape memory alloys since their stable output is always expected. Some types of the thermomechanical cycling or training are reported to be effective to improve the shape memory alloy performance when the slip deformations are suppressed in the work-hardened parent phase and the microstructures favorable to the applied stress are created [15]. The two-way shape memory effect, the reversible shape change by only thermal cycling without any applied 
Fumihito NishimuRA, Noriko Watanabe and Kikuaki TANAKA

stress, is observed after the sufficient number of thermomechanical cycling, in which the internal residual stress generated during cycling plays an important role [16-18].

In order to design the large Fe-based shape memory devices, not only the thermomechanical behavior under the complex stress states but also the cyclic behavior must be clarified. Recently many researchers contribute to construct the constitutive theories for shape memory alloys [19-24]. Sufficient and sound experimental data are urgently necessary for this purpose.

In this paper the thermomechanical hysteretic behavior is investigated experimentally in an polycrystalline Fe-9\% Cr-5\%Ni-14\%Mn-6\%Si shape memory alloy under thermomechanical cyclic loading. The tensile and/or compressive stress are loaded to study the effect of the direction of the applied stress. The change in shape and the drift as a whole of the stress-strain-temperature loop during cycling, are the point to be noted. The evolution of the residual strain and the transformation strain is, together with the change in the martensite start stress, interpreted by means of the metallurgical observations in the Fe-based shape memory alloys $[3-5,25,26]$.

\section{EXPERIMENTAL PROCEDURE}

The shape memory alloy used in the present study, Fe-9\%Cr-5\%Ni-14\%Mn-6\%Si polycrystal, was prepared by vacuum induction melting [27]. The specimens in Fig. $1,6 \mathrm{~mm}$ in diameter with $20 \mathrm{~mm}$ in gauge length, were machined from the hot-rolled plate which had been homogenized at $1323 \mathrm{~K}$ for $3600 \mathrm{~s}$.

Tests were carried out with a servo-hydraulic thermal fatigue testing machine (SHIMADZU, EHF-ED5/TD0510L) equipped with a high frequency induction heater, with which both the mechanical and thermal cyclic loads were applicable independently in any form of time history. The displacement was measured with a differential transformer, whereas the temperature of the specimen was detected with a platinum-platinum rhodium thermocouple spot-welded at the center in the gauge length. All experimental procedures were monitored and controlled by a personal computer.

During the whole tests in this paper, the loading and unloading rates were kept constant at about $3 \mathrm{MPa} / \mathrm{s}$, whereas the heating rate was maintained at about $3 \mathrm{~K} / \mathrm{s}$. The cooling rate was also kept constant at the same value as close as possible by controlling the pressurized air flow to the specimen.

Two types of specimen were supplied to the test; the untrained, as machined specimens and the trained

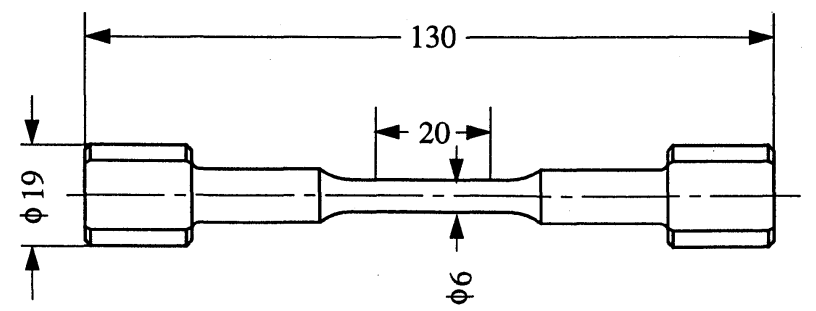

Fig.1. Test specimen. specimens. The latter specimens were, prior to the tests subjected to 4 times of the following thermomechanical training: An isothermal mechanical loading in tension up to $4 \%$ at the room temperature (RT, $303 \mathrm{~K}$ ) and unloading followed by a thermal heating up to $873 \mathrm{~K}$, holding there for $600 \mathrm{~s}$ and cooling down to RT.

\section{RESULTS AND DISCUSSIONS}

\subsection{Tensile Stress Controlled Loading}

The untrained test specimen was subjected to 50 cycles of thermomechanical loading, one cycle of which was composed of an isothermal mechanical loading at the hold temperature $T_{h}=\mathrm{RT}$ up to the maximum stress $\sigma_{\max }^{+}=$ $350 \mathrm{MPa}$ and unloading down to the hold stress $\sigma_{h}=0$ $\mathrm{MPa}$ and a subsequent isostatic heating under the hold stress $\sigma_{h}$ up to $T_{\max }=873 \mathrm{~K}$ and cooling down to RT. Since the maximum temperature is higher than the austenite finish temperature, the reverse transformation during heating process always completes. The martensitic transformation in the isothermal mechanical run does not finish before the fracture of the specimen.

Figure 2 shows the stress-strain-temperature hysteretic behavior during cycling. To avoid the complexity in reading the figure, only the hysteresis loops for the number of cycles $N=1,2, \ldots, 5$ and $10,20, \ldots, 50$ are plotted. The hysteresis loops of the present alloy continue shifting to the higher strain side with keeping its shape unchanged.

The hysteresis loop in Ti-Ni [28-30] and Cu-based $[31,32]$ shape memory alloys, on the contrary, exhibits the change in shape and the convergence to the stationary limit loop which is on the higher strain side compared to the initial loop. This shift and convergence of the

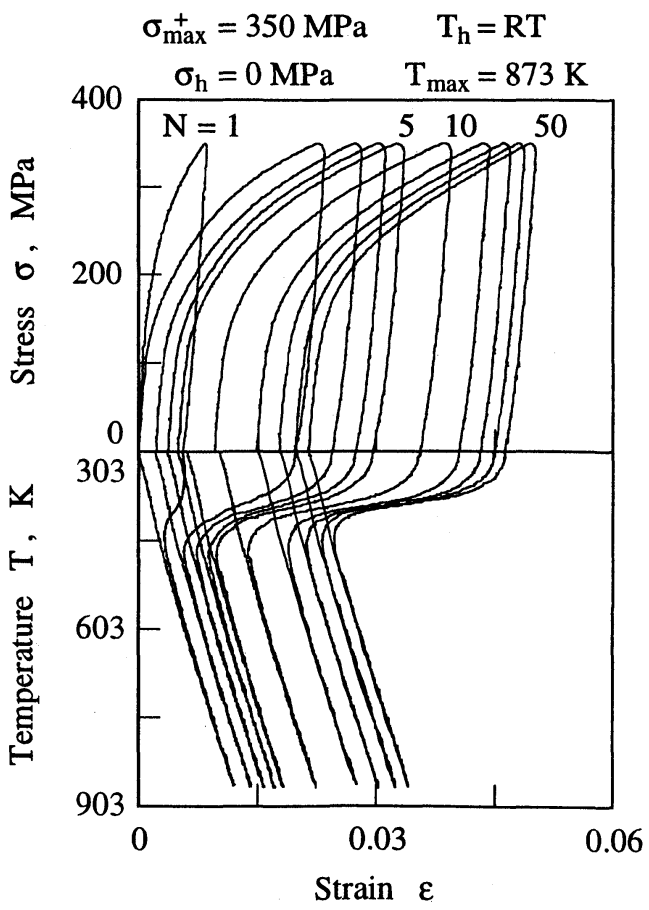

Fig.2. Stress-strain-temperature hystereses; Tensile loading. 
hysteresis loop are attributed to the pileup of dislocations around the microscopic defects dispersed in the material, caused by the repeated sweeps of the phase interface between the martensite and the parent phases. The dislocations induce the microscopic residual stress, which stabilizes the martensite, resulting eventually in the residual strain increase. When the accumulation of the dislocations saturates, the hysteresis loop converges to the limit loop.

The $\varepsilon$ martensite plate observed in the Fe-based shape memory alloys is understood as the extended dislocations, which are composed of the stacking faults with the partial dislocations attached at both ends [3-5]. During the thermomechanical loading the $\varepsilon$ martensite changes its length so that the applied driving force balances with the expulsive force by the partial dislocations and the attractive force inside the stacking faults. The $\varepsilon$ martensite plates with different orientation collide in this process with each other to form the tangled perfect dislocations, which disappear only when heated above the recrystallization temperature. The perfect dislocations accumulate in the process of loading, or cycle by cycle in the case of cyclic loading and become the origin of the increase of the residual strain.

The metallurgical observation in Fe-based shape memory alloys has also revealed that the local back

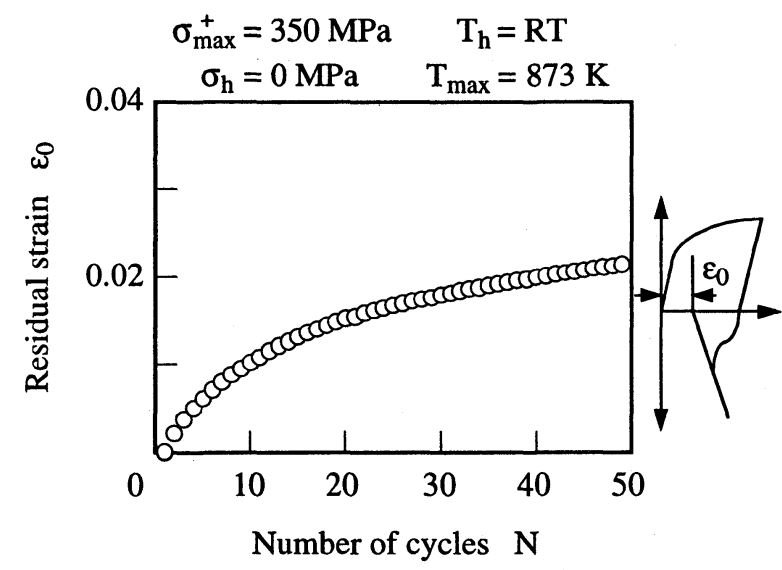

Fig.3. Increase in residual strain.

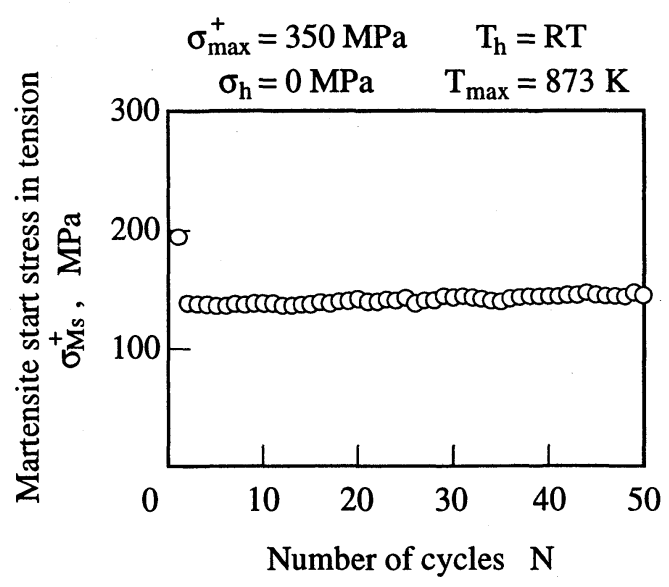

Fig.4. Martensite start stress. stress, which is generated due to the strain misfit between the martensite and the parent phases, guarantees the shape reversibility between the two phases [25]. While the martensitic transformation progresses, the selfaccommodation takes place due to the widening of the martensite plates. This decreases the back stress, and the shape reversibility as well. The shape irreversibility may be the origin of the continuous accumulation of the residual strain in the present alloy as observed in Fig.3. The change per cycle of the residual strain $\varepsilon_{0}$, defined in the figure as the remaining strain after reverse transformation, becomes sharply smaller with the number of cycles. The curve looks to tend to a linear line. This suggests that the increase in the residual strain comes not only from the above-mentioned shape irreversibility but also from the accumulation of the perfect dislocations. After 50 cycles of loading, the residual strain reaches $2.12 \%$ but still increases by $1.4 \times 10^{-2} \%$ per cycle, which corresponds to $0.54 \%$ of the width of the strain amplitude.

The martensite start stress $\sigma_{M s}^{+}$, determined as a $0.05 \%$ proof stress, remains, as shown in Fig.4, almost constant during cycling except at the first cycle. The martensite start stress at $N=1$ is $\sigma_{M s}^{+}=194.3 \mathrm{MPa}$, which is larger by about $50 \mathrm{MPa}$ than the values in the subsequent cycles. This result is different from the observations in Ti-Ni alloy [28-30] and $\mathrm{Cu}$-based alloys [31,32], exhibiting the gradual decrease in the martensite start stress during cyclic loading and its convergence to a limit value. The phenomenon can be attributed to the perfect dislocation pileup during cycling. The microscopic residual stress is induced as a result, and it makes the start of the martensitic transformation easier, meaning the decrease in the martensite start stress. So the stress-strain curve shifts to the lower stress side with cycling. Figure 4, however, indicates that in the present alloy little amount of local residual stress is generated during cycling. This does not mean that the dislocation pileup is not observed during cycling, but the residual stress induced due to dislocation pileup does not assist the martensitic transformation.

The transformation strain $\varepsilon_{T}^{+}$in Fig.5, which is induced by the martensitic transformation during

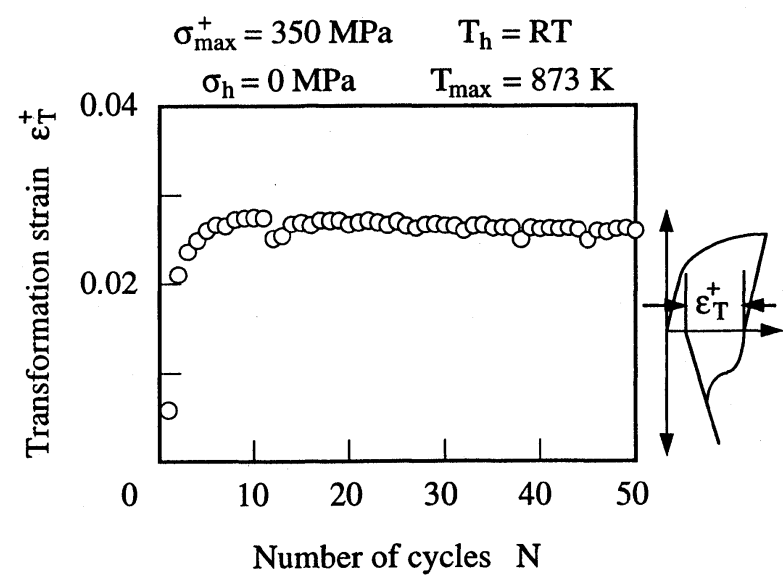

Fig.5. Transformation strain during cycling. 
isothermal mechanical loading and has a value of $0.58 \%$ at $N=1$, jumps up to $2.11 \%$ at the second cycle and rapidly increases to its maximum value $2.75 \%$ at $N=9$. Then it slowly decreases to $2.60 \%$ at $N=50$.

If the martensite start stress decreases with cycling, the difference between the maximum stress and the martensite start stress increases with cycling, resulting in the increase in the transformation strain. In the present alloy, however, the change in the transformation strain should be attributed to another reasons when observing almost no change in the martensite start stress in Fig.4. As the martensitic transformation progresses, the microstructures in the alloy change from the initial orientationindependent state since the variants favorable to the applied stress are formed. This microscopic structural change explains the sharp increase of the transformation strain at the early stage of cycling [17].

\subsection{Compressive Stress Controlled Loading}

Similar experiment was carried out in compressive loading using the untrained specimens The specimen was subjected to 50 cycles of the following thermomechanical loading: A mechanical run down to $\sigma_{\max }^{-}=-350 \mathrm{MPa}$ at $T_{h}=$ RT followed by a thermal run up to $T_{\max }=873 \mathrm{~K}$ under $\sigma_{h}=0 \mathrm{MPa}$. Figure 6 shows the hysteresis loops for the number of cycles $N=1,2, \ldots, 5$ and $10,20, \ldots$, 50. Similar to the tensile loading in Fig.2, the hysteresis loops shift to the compressive strain side with keeping their shape unchanged except at the first cycle.

The martensite start stress in compression $\sigma_{M s}^{-}$, determined as a $0.05 \%$ proof stress and displayed in Fig. 7 , remains almost constant except at the first cycle, at which $\sigma_{M s}^{-}=-230.4 \mathrm{MPa}$ is about $50 \mathrm{MPa}$ higher than the values in the subsequent cycling. It should be noted

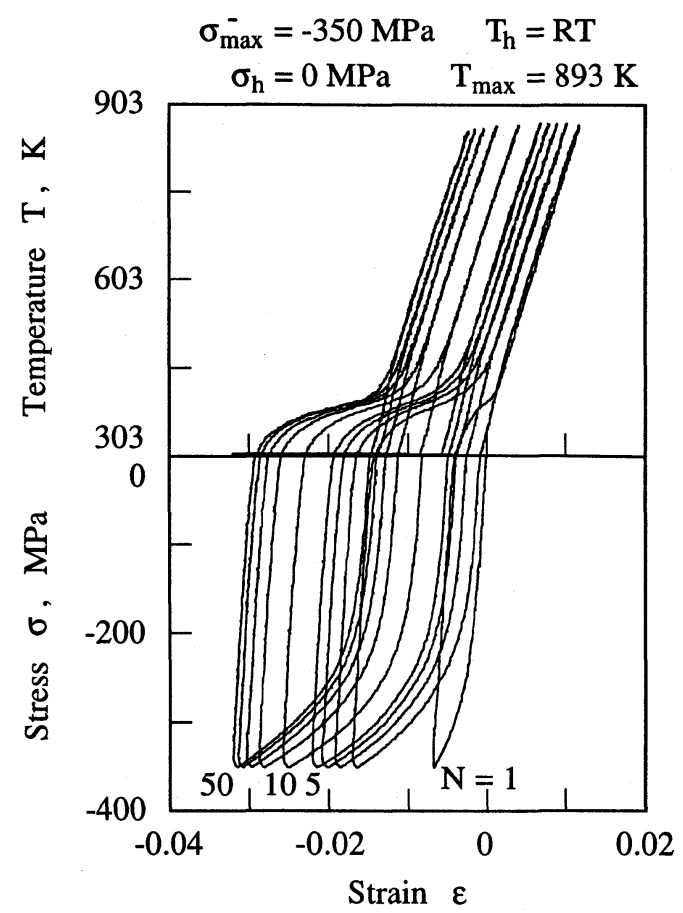

Fig.6. Stress-strain-temperature hystereses; Compressive loading. that the magnitude of this drop is nearly the same as observed in the tensile cycling (cf. Fig.4).

The change in the transformation strain in compression $\overline{\varepsilon_{T}}$ is shown in Fig.8. The extent of martensitic transformation per cycle is smaller in compression than in tension since $\sigma_{M s}^{+}<\left|\sigma_{M s}^{-}\right|$. So the magnitude of the transformation strain in compression is smaller than in tension, $\left|\varepsilon_{T}^{-}\right|<\varepsilon_{T}^{+}$. Similar to the results in tension a sudden change in transformation strain from $-0.44 \%$ at $N=1$ to $-1.43 \%$ at $N=2$ is followed by a slight increase to the maximum value of $-1.59 \%$ at $N=$ 11. At the end of cycling the transformation strain reduces to $-1.51 \%$. The shift of the hysteresis loop is illustrated in Fig. 9 as the evolution of the residual strain $\varepsilon_{0}$, which changes almost linearly after a rapid increase at the early stage of cycling. In the linear region the residual strain increases by $-6 \times 10^{-3} \%$ per cycle, which corresponds to $0.4 \%$ of the transformation strain. The compressive residual strain after 50 cycles of loading reaches $-1.48 \%$. A stable limit loop is not attained in the compressive cycling, at least by $N=50$.

Comparison of the total amount of the residual strain in tension and compression reveals that the larger residual strain is induced when the larger transformation strain is produced. This agrees with such a metallurgical observation that the martensite plates widen its width to

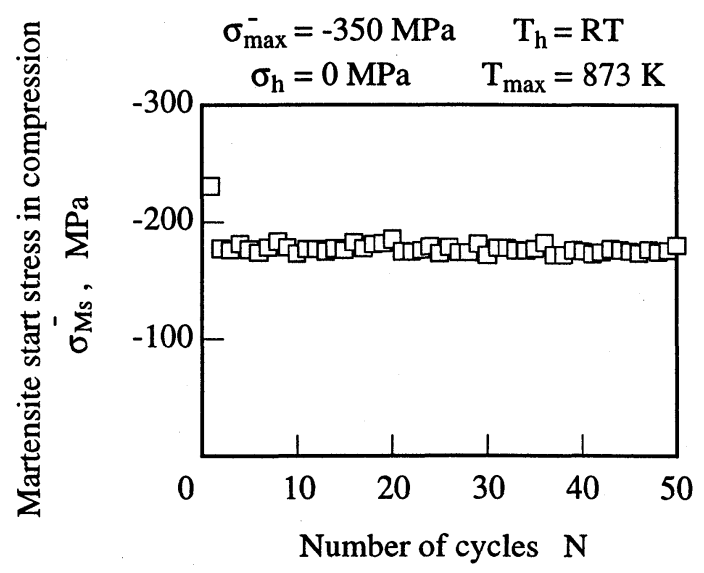

Fig.7. Martensite start stress.

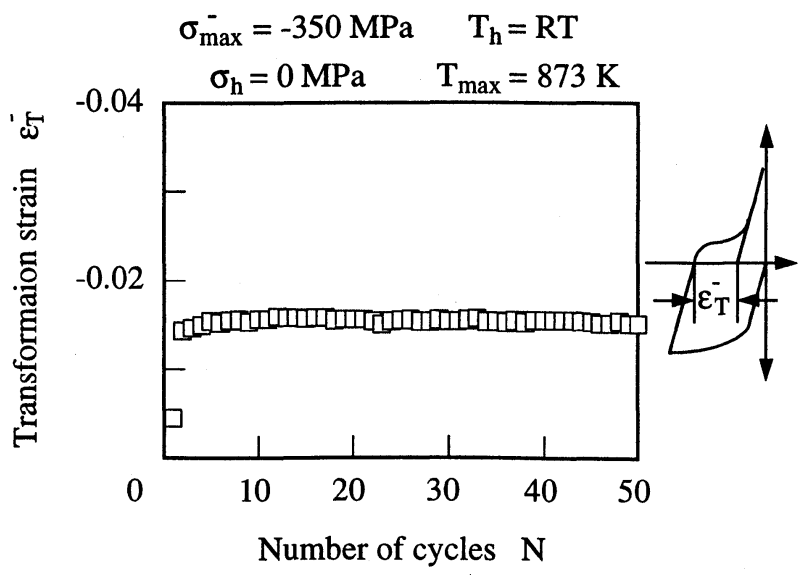

Fig.8. Transformation strain. 
reduce the shape reversibility in the reverse transformation. The effect becomes dominant as the martensitic transformation progresses [25]. In addition, the larger extent of the transformation in each cycle means that the phase interfaces sweep a lager area in the alloy, resulting in the formation of the larger amount of perfect dislocations, which eventually produces the larger amount of the residual strain.

\subsection{Strain Controlled Loading}

The cyclic behavior was also investigated under the strain controlled loading. The trained specimen was subjected to 100 cycles of the following thermomechanical loading: An isothermal loading at $T_{h}=$ RT to maximum strain $\varepsilon_{\text {max }}=2$ or $3 \%$ and unloading to $\sigma_{h}=0 \mathrm{MPa}$ are followed by an isostatic heating under $\sigma_{h}=0 \mathrm{MPa}$ up to $T_{\max }=873 \mathrm{~K}$ and cooling down to RT.

Figure 10 plots the hysteresis loops for $\varepsilon_{\max }=3 \%$ at the number of cycles $N=1,10,20, \ldots, 100$. Like the results in the stress controlled cycling, the residual strain $\varepsilon_{0}$ is accumulated cycle by cycle since the transformation strain induced during mechanical loading is not fully recovered. This leads to the shift of hysteresis loop to the higher strain side.

Figure 11 shows the evolution of the residual strain during cyclic loading. The residual strain for $\varepsilon_{\max }=3 \%$ increases rapidly at the early stage of cycling, and at around $N=15$ the curve becomes almost linear. In the first 15 cycles about $50 \%$ of the residual strain accumulated after 100 cycles of loading is produced. On the other hand, for the case of $\varepsilon_{\max }=2 \%$, the residual strain increases linearly from the very beginning of cycling. The fact that both curves have nearly the same slope in the linear part suggests the following conclusion: There are two mechanisms of producing $\varepsilon_{0}$, the one of which works at the early stage of cycling under the large $\varepsilon_{\text {max }}$. The other mechanism becomes dominant as the cycle progresses, which is active regardless of the magnitude of $\varepsilon_{\max }$.

In the case of strain controlled loading with a constant $\varepsilon_{\text {max }}$, the transformation strain $\varepsilon_{T}=\varepsilon_{\text {max }}-\varepsilon_{0}$ decreases cycle by cycle due to constant increase in the residual strain. This fits to the data in Fig.12 showing the

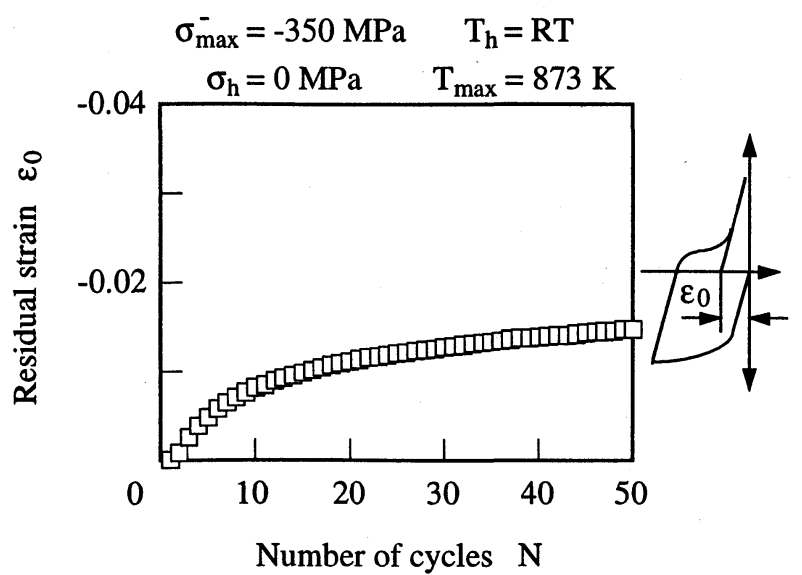

Fig.9. Residual strain. maximum stress $\sigma_{\max }$, which is a measure to estimate the transformation strain, decreases cycle by cycle. The rapid decrease in $\sigma_{\max }$ at the early stage of cycling is followed by a linear part in the case of $\varepsilon_{\max }=3 \%$, whereas in the case of $\varepsilon_{\text {max }}=2 \% \sigma_{\max }$ decreases almost linearly from the beginning. Corresponding to the continual increase in the residual strain, the decrease in the maximum stress also continues even after 100 cycles of loading.

The evolution of the residual strain is displayed in Fig. 13 during both the strain controlled loading of $\varepsilon_{\text {max }}=$ $3 \%$ and the stress controlled loading of $\sigma_{\max }=350 \mathrm{MPa}$. During training prior to the test, a certain amount of residual strain is accumulated to form the work-hardened specimen. So the residual strain increases almost linearly from the beginning in the trained specimen. On the other hand, some 20 cycles of loading is necessary to realize the

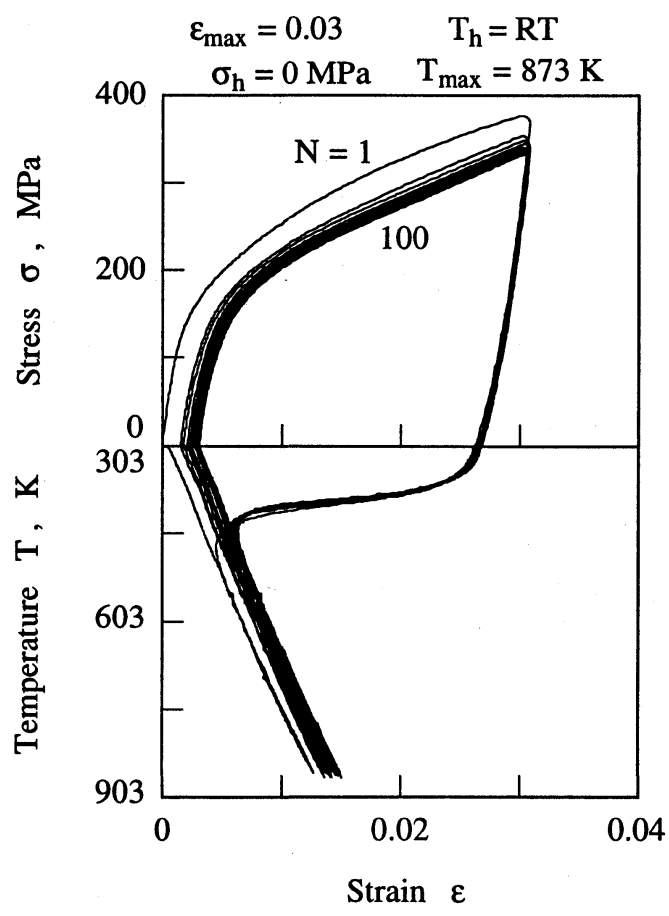

Fig.10. Stress-strain-temperature hystereses; Strain controlled loading.

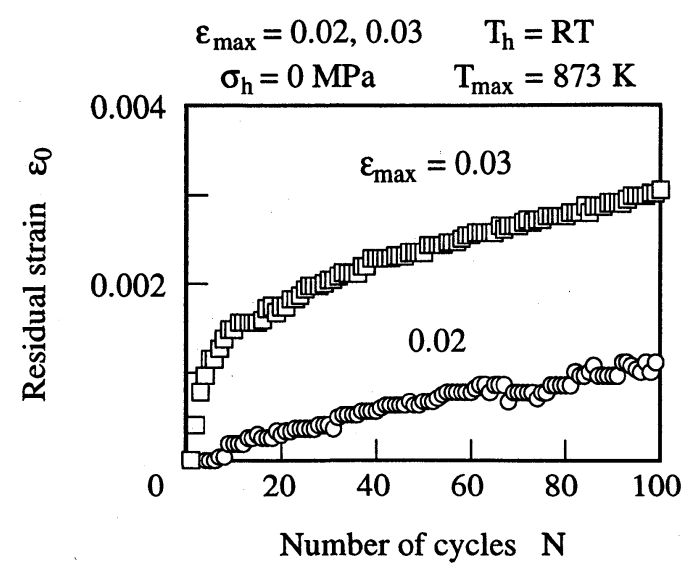

Fig.11. Increase in residual strain. 
same state in the untrained specimen. The rapid increase in the residual strain is observed during this "training." The fact that the slopes of the linear part of the two curves are nearly the same again suggests that the second deformation mechanism mentioned above works after being trained.

Figure 13 clearly shows that the larger amount of the residual strain is induced in the stress controlled tests than in the strain controlled test. The deformation mechanisms in the present alloy are now discussed in detail by referring to the metallurgical observations in the Fe-based alloys $[10,11,25]$. The residual strain increases only slightly under the low applied stress since the martensitic transformation progresses a little, resulting in a small accumulation of the perfect dislocations. The residual strain progresses due to the accumulated perfect dislocations. This is the case of $\varepsilon_{\max }=2 \%$ in Fig. 11 . If the stress exceeds a critical value, about $350 \mathrm{MPa}$ in the present case, the wider martensite plates must be formed, which become the cause of the shape irreversibility in the reverse transformation. This second mechanism of producing the residual strain seems to be active at the early stage of cycling in the case of $\varepsilon_{\max }=3$ $\%$ till $\sigma_{\max }$ becomes $350 \mathrm{MPa}$ at around $N=15$ (cf. Fig.12). The change in the mode of $\varepsilon_{0}$-evolution is already observed in Fig. 11 at around $N=15$. From this cycle on the first mechanism must become dominate to produce a constant growth of $\varepsilon_{0}$ with nearly the same slope as in the case of $\varepsilon_{\max }=2 \%$.

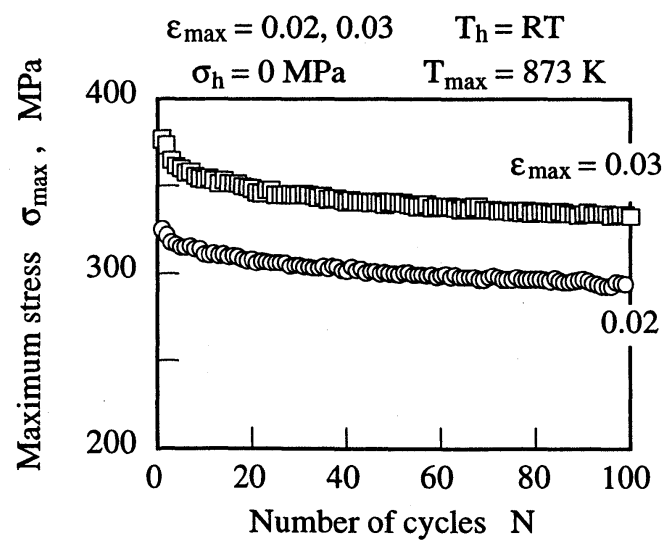

Fig.12. Stress at maximum strain.

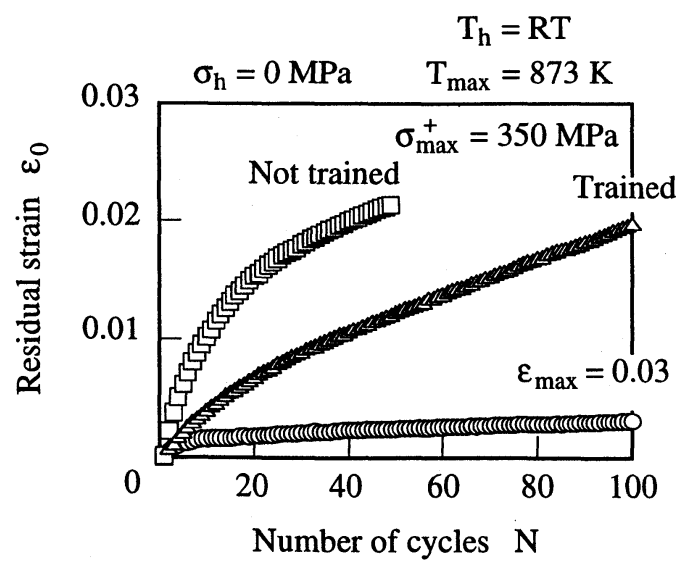

Fig.13. Residual strain.

\subsection{Alternating Stress Controlled Loading}

Using the untrained specimen, the hysteretic behavior during alternating stress controlled loading was investigated. The specimen was first mechanically loaded at the constant hold temperature $T_{h}=\mathrm{RT}$ up to the maximum tensile stress $\sigma_{\text {max }}^{+}=350 \mathrm{MPa}$ and unloaded down to the stress-free state $\sigma_{h}=0 \mathrm{MPa}$. After isostatic heating/cooling under $\sigma_{h}=0 \mathrm{MPa}$ between the maximum temperature $T_{\max }=873 \mathrm{~K}$ and the hold temperature $T_{h}=$ $\mathrm{RT}$, the isothermal compression was carried out at $T_{h}=$ RT down to the maximum compressive stress $\sigma_{\max }^{-}=$ $-350 \mathrm{MPa}$. The unloading was followed by the isostatic heating under free-stress up to $T_{\max }=873 \mathrm{~K}$ and the subsequent cooling down to $T_{h}=\mathrm{RT}$. The martensite phase is induced in tension at the odd-numbered cycles, and in compression at the even-numbered cycles.

Figure 14 gives the stress-strain-temperature hystereses at the numbers of cycles $N=1,2,9,10,19,20, \ldots, 199$, 200. Similar to the previous results using the untrained specimen, a small hysteresis is observed in the first cycle. A compressive residual strain is, as a result, produced at the second cycle. From then on the hysteresis loops shift to the higher strain side cycle by cycle. The crowded hystereses illustrated in Fig. 14 stem from a little change in the residual strain compared to the case of the pulsating stress controlled test in Figs. 2 and 6.

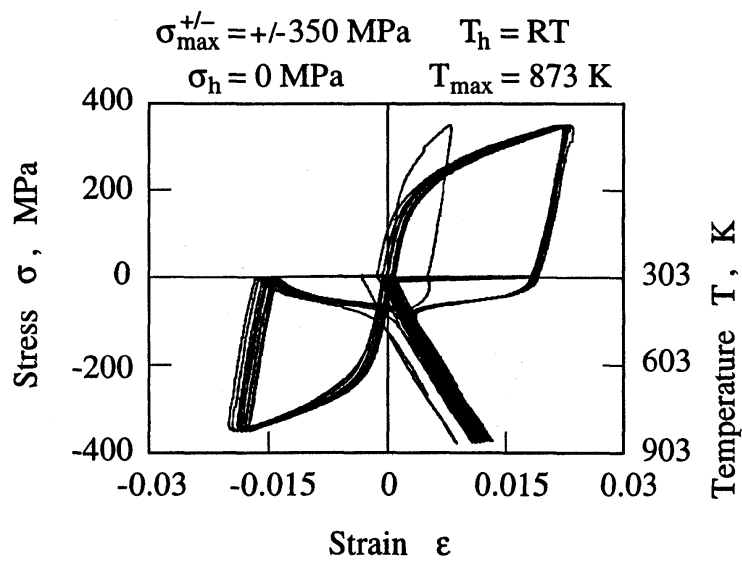

Fig.14. Stress-strain-temperature hystereses; Alternating stress loading.

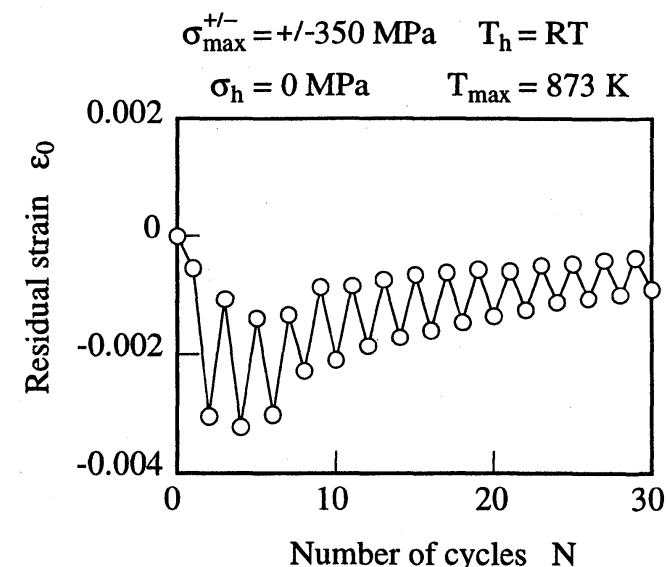

Fig.15. Residual strain. 
Figure 15 plots the residual strain at the early stage of cycling. Neglecting the negative residual strain in the first martensitic transformation in tension, which stems from the ill specimen setting, the direction of the residual strain is the same as the direction of loading. The overall value of the residual strain increases with cycling, and reaches $7.8 \times 10^{-2} \%$ after 200 cycles of loading, but it still continues increasing by $5 \times 10^{-4} \%$ per cycle as shown in Fig. 16.

Figure 16 also displays the difference in evolution of the residual strain in the three different tests. The upward triangles represent the residual strain in tension whereas the downward ones represent that in compression. The squares obtained by summing the two curves for the pulsating stress controlled tests exhibits a similar characteristics of the curve plotted with the open circles for the alternating stress controlled test, but its amount is almost 18 times larger. At each cycle the variant has to be switched from the previous arrangement to the new ones favorable to the present applied stress having the fully opposite direction. This cyclic rearrangement of the variants reduced the residual strain.

The martensite start stress during cycling is given in Fig.17. The martensite start stress stays, except at the first cycle, nearly unchanged during the alternating stress controlled loading in both tensile and compressive branches. The data also reveal that the martensite start stress in tension has almost the same values as in the case of the tensile stress controlled loading which are replotted from Fig.4. The same is true for the martensite start stress in compression.

The data of the transformation strain in Fig.18 show completely different characteristics, depending strongly on the tests. The transformation strain in the alternating stress controlled loading exhibits the maxima; $2.17 \%$ in tension at $N=7$ and $-1.81 \%$ in compression at $N=8$. After that the transformation strain changes slightly, and from $N=50$ on it remains almost constant. The value of the residual strain in tension is larger than in compression, irrelevant to the pulsating and alternating stress controlled loading. This can be attributed to the asymmetry of the martensite start lines in tension and compression with respect to the stress [33]. For the same value of $\sigma_{\max }^{+}$and $\sigma_{\max }^{-}$, as in the present alternating

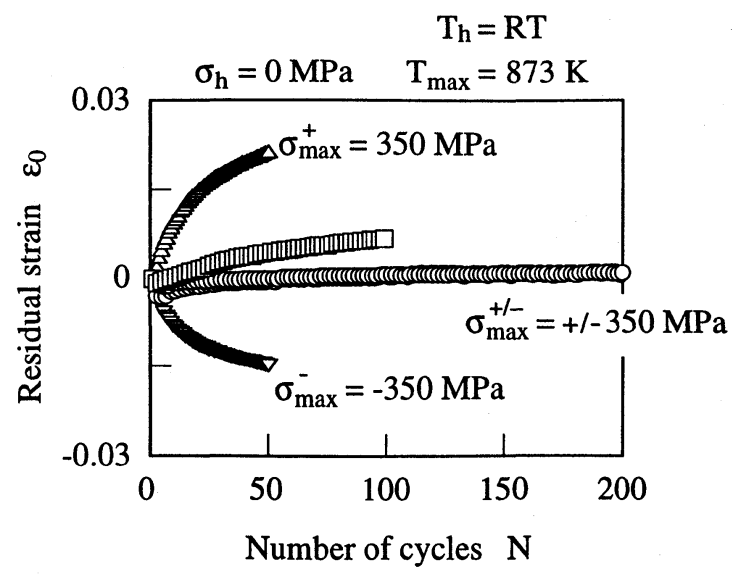

Fig.16. Residual strains. stress controlled test, the extent of martensitic transformation in tension is larger than in compression, producing the larger amount of transformation strain after tensile loading than after compressive loading.

The different values of the transformation strain are observed in the alternating stress controlled test and the pulsating stress controlled test in tension. This phenomenon can be explained from the fact that the formation of the variants favorable to the applied stress is easier in the pulsating stress controlled test than in the alternating stress controlled test. The behavior on the compressive side, in which, despite the clear metallurgical reason explained above, the transformation strain has nearly the same values in both the pulsating stress controlled test and the alternating stress controlled test, is unclear.

\section{CONCLUDING REMARKS}

The thermomechanical behavior in an Fe-based polycrystalline shape memory alloy is investigated under tensile and/or compressive cyclic thermomechanical loading. The stress-induced martensitic transformation and the temperature-induced reverse transformation take place repeatedly during thermomechanical cycling. The stress-strain-temperature hysteresis loop shift to the high strain side during both tensile and compressive pulsating

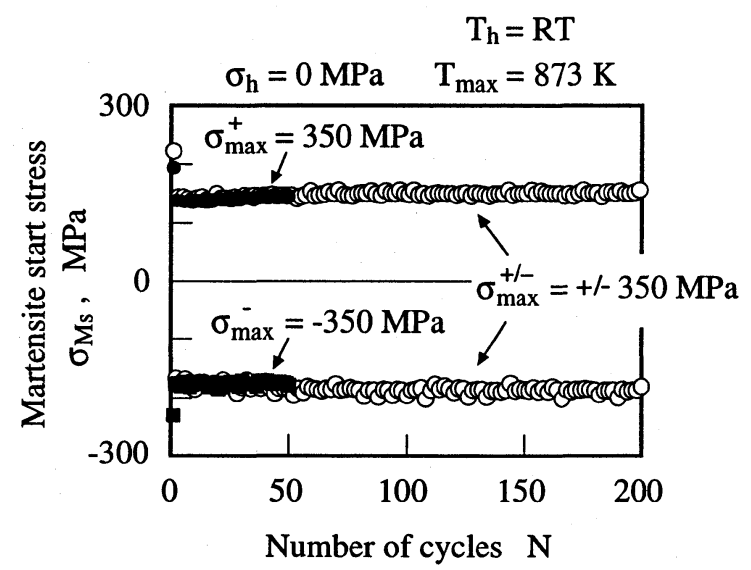

Fig.17. Martensite start stresses.

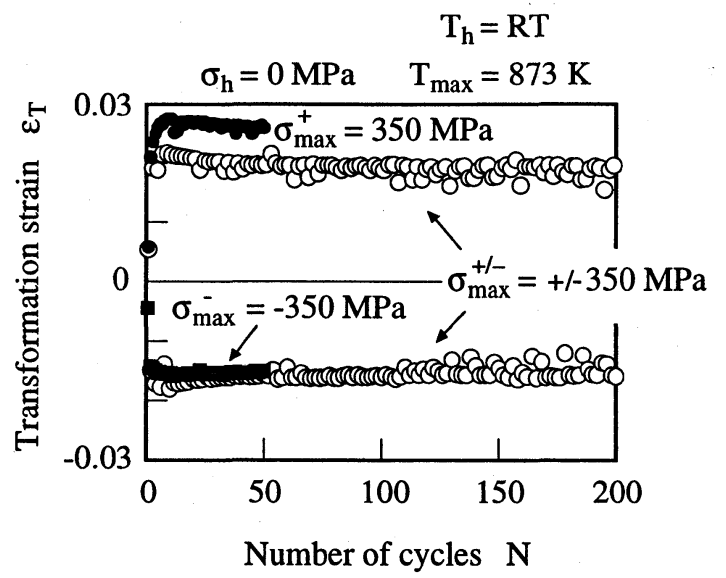

Fig.18. Transformation strains. 
stress cycling. The shift under the alternating stress cycling is not a simple superposition of the results in pulsating stress cycling in tension and compression. Contrary to the results in $\mathrm{Ti}-\mathrm{Ni}$ and $\mathrm{Cu}$-based shape memory alloys, the shape of the loop stays unchanged and the loop does not converge to a limit loop, at least by 100 cycles investigated.

Two microscopic mechanisms seem to be responsible to the evolution of the residual strain, which control the shift of the hysteresis loop and the transformation strain which governs the size of hysteresis loop. The first mechanism always seems to work at the early stage of cycling, the effect of which is large with large strain or stress applied. The phenomenon is attributed to the shape irreversibility in the reverse transformation, which stems from the reduction of the back stress during widening of the martensite plates. The second mechanism, being typical in the Fe-base alloys, becomes dominant as the cycle progresses. The metallurgical cause of this effect is tangled perfect dislocations formed at the place of collision of the $\varepsilon$ martensite plates with different orientation.

The alloy behavior under the alternating stress cycling should be explained by the results under the pulsating stress cycling with the help of more detailed metallurgical observation of the variants favorable to the applied stress.

Acknowledgment - The authors wish to thank Prof. H. Inagaki, Shonan Institute of Technology, for his constructive criticism concerning an earlier version of the manuscript. Part of this work was financially supported by the Special Research Fund/ Tokyo Metropolitan Government as well as by the Grant-in-Aid for Scientific Research (No.98650117) through the Ministry of Education, Science and Culture, Japan.

\section{REFERENCES}

1. A. Sato, E. Chishima, K. Soma, T. Mori, Acta Metall., 30 (1982) 1177.

2. H. Otsuka, H. Yamada, T. Maruyama, H. Tanahashi, S. Matsuda and M. Murakami, ISIJ International, 30 (1990) 674.

3. H. Inagaki, Z. Metallkd., 83 (1992) 90.

4. H. Inagaki and K. Inoue, Z. Metallkd., 85 (1994) 790.

5. H. Inagaki, Z. Metallkd., 86 (1995) 35.

6. D.Z. Liu, W.X. Liu and F.Y. Gong, J. Physique, Coll.8, suppl. J. Physique III, 5 (1995) 1241.

7. T.W. Duerig, K.N. Melton, D. Stökel and C.M. Wayman eds., Engineering Aspects of Shape Memory Alloys, Butterworth-Heinemann, London (1990).

8. S. Eucken ed., Progress in Shape Memory Alloys, DGM Informationsgesellschaft Verlag, Oberursel (1992).

9. T. Maki and I. Tamura, Proc. ICOMAT 86 (1986) 389.

10. T. Kikuchi, S. Kajiwara and Y. Tomota, J. Physique, Coll.8, suppl. J. Physique III, 5 (1995) 445.
11. H. Ohtsuka, S. Kajiwara, T. Kikuchi, T. Ishihara and K. Nagai, J. Physique, Coll.8, suppl. J. Physique III, 5 (1995) 451.

12. J.C. $\mathrm{Li}$ and G.S. Ansell, Metall. Trans., $14 \mathrm{~A}$ (1983) 1293.

13. K. Tsuzaki, M. Ikegami, Y. Tomota, Y. Kurokawa, W. Nakagawara and T. Maki, Materials Trans., JIM, 33 (1992) 263.

14. K. Tanaka, T. Hayashi, F. Nishimura and H. Tobushi, J. Materials Engng Performance, 3 (1994) 135.

15. D.P. Dunne and H. Li, J. Physique, Coll.8, suppl. J. Physique III, 5 (1995) 415.

16. J. Pons, M. Sade, F.C. Lovey and E. Cesari, Materials Trans., JIM, 34 (1993) 888.

17. A. Amengual, E. Cesari and J. Pons, J. Physique, Coll.8, suppl. J. Physique III, 5 (1995) 871.

18. H. Tobushi, P.H. Lin, T. Hattori and M. Makita, JSME Int. J., Ser.A, 38 (1995) 59.

19. D.C. Lagoudas, Z. Bo and M.A. Qidwai, Mech. Composite Mater. Struct., 3 (1996) 153.

20. Y. Ivshin and T.J. Pence, Proc. ICOMAT 92 (1993) 389.

21. Q.P. Sun and C. Lexcellent, J. Physique, Coll.1, suppl. J. Physique III, 6 (1996) 367.

22. K. Tanaka, F. Nishimura, T. Hayashi, H. Tobushi and C. Lexcellent, Mech. Materials, 19 (1995) 281.

23. K. Tanaka, E.R. Oberaigner and F.D. Fischer, Mechanics of Phase Transformations and Shape Memory Alloys, ASME (1994) 151.

24. K. Tanaka, D. Hasegawa, H.J. Böhm and F.D. Fischer, Mater. Sci. Res. Int., 1 (1995) 23.

25. K. Tsuzaki, Y. Natsume and T. Maki, J. Physique, Coll.8, suppl. J. Physique III, 5 (1995) 409.

26. T. Maki and K. Tsuzaki, Proc. ICOMAT 92 (1993) 1151.

27. Y. Moriya, H. Kimura, S. Ishizaki, S. Hashizume, S. Suzuki, H. Suzuki and T. Sampei, Proc. European Symp. Martensitic Transformation and Shape Memory Properties (1991)

28. B. Strnadel, S. Ohashi, H. Ohtsuka, T. Ishihara and S. Miyazaki, Mater. Sci. Eng. A, 202 (1995) 148.

29. H. Tobushi, H. Iwanaga, K. Tanaka, T. Hori and T. Sawada, Contin. Mech. Thermodyn., 3 (1991) 79.

30. H. Tobushi, A. Ikai, S. Yamada, K. Tanaka and C. Lexcellent, J. Physique, Coll.1, suppl. J. Physique III, 6 (1996) 385.

31. G. Bourbon, C. Lexcellent and S. Leclerc, J. Physique, Coll.8, suppl. J. Physique III, 5 (1995) 221.

32. M. Tokuda, P. Sittner, M. Takakura and Y. Men, Mater. Sci. Res. Int., 1 (1995) 260.

33. F. Nishimura, N. Watanabe and K. Tanaka, Mater. Sci. Eng. A, 221 (1996) 134. 